Original Article

\title{
Targeting pain catastrophization in patients with fibromyalgia using virtual reality exposure therapy: a proof-of-concept study
}

\author{
Linzette Deidrè Morris ${ }^{1)^{*}}$, Quinette Abegail Louw ${ }^{1)}$, Karen Anne Grimmer ${ }^{1,2)}$, \\ ERnesta Meintues ${ }^{3)}$ \\ 1) Division of Physiotherapy, Department of Interdisciplinary Health Sciences, Faculty of Medicine \\ and Health Sciences, Stellenbosch University: Tygerberg, South Africa \\ 2) International Centre for Allied Health Evidence (iCAHE), University of South Australia, Australia \\ 3) MRC/UCT Medical Imaging Research Unit, Faculty of Health Sciences, University of Cape Town, \\ South Africa
}

\begin{abstract}
Purpose] Pain catastrophizing is a key predictor of poor compliance to exercises among patients with fibromyalgia syndrome. Alteration of pain catastrophizing in this group is thus warranted. This study aimed to provide proof-of-concept of a novel virtual reality exposure therapy program as treatment for exercise-related pain catastrophizing in patients with fibromyalgia syndrome. [Subjects and Methods] An exploratory, case-controlled study was conducted (fibromyalgia syndrome group and matched control group). Functional magnetic resonance imaging was used to acquire neural correlates. The functional magnetic resonance imaging task consisted of two stimuli: active (exercise activity visuals) and passive (relaxing visuals). Structural images and blood-oxygenation-leveldependent contrasts were acquired for the conditions and compared within subjects/groups and between groups. Statistic images were thresholded using corrected clusters (determined by $Z>2.3$; level of significance: 0.05 ). [Results] Thirteen fibromyalgia syndrome subjects and nine healthy matched controls were included. The right inferior frontal gyrus, right middle frontal gyrus, right posterior cerebellum, left thalamus, and left supramarginal gyrus were activated in the fibromyalgia syndrome subjects. [Conclusion] The study results provide preliminary proof indicating that exposing patients with fibromyalgia syndrome to visuals of exercises elicits neurophysiological changes in functional brain areas associated with pain catastrophization and add to the current body of knowledge regarding the possibility of objectively identifying cognitive behavioral strategies like pain catastrophization. Key words: Fibromyalgia, Pain catastrophizing, Exercise
\end{abstract}

(This article was submitted Jul. 2, 2015, and was accepted Aug. 19, 2015)

\section{INTRODUCTION}

Consistent evidence from meta-analyses and clinical practice guidelines strongly indicate that exercise therapy should be a key component in the management of fibromyalgia syndrome (FMS), a complex chronic pain condition $^{1-4)}$. However, the implementation of exercise therapy as a management strategy for FMS in practice is significantly hampered by poor compliance ${ }^{5-11)}$. A key predictor of poor compliance often displayed among patients with FMS has recently been identified as pain catastrophization. A cognitive strategy, pain catastrophization is broadly defined as "an exaggerated negative orientation towards actual or anticipated pain experiences" that significantly contributes

\footnotetext{
*Corresponding author. Linzette Deidré Morris (E-mail:
} ldmorris@sun.ac.za)

C2015 The Society of Physical Therapy Science. Published by IPEC Inc. This is an open-access article distributed under the terms of the Creative Commons Attribution Non-Commercial No Derivatives (by-ncnd) License $<$ http://creativecommons.org/licenses/by-nc-nd/3.0/> . to the maintenance of pain ${ }^{12-16)}$. Currently, the role of pain catastrophization is believed to be more pronounced in FMS than in other rheumatologic chronic pain condition and is recognized as a barrier to the healthy reestablishment of psychological and physical functioning among patients with FMS ${ }^{13-17)}$. Of concern is that in patients with FMS, the presence of pain catastrophization leads to fear-avoidance behaviours that often result in attrition from regular physical activity ${ }^{13)}$. Inactivity is particularly detrimental in FMS and typically leads to further complications such as deconditioning of the musculoskeletal system, increased pain, increased fatigue and functional disability ${ }^{6,16)}$. Fundamentally, poor compliance with exercise and physical activity among patients with FMS is the primary factor contributing to the chronicity and accelerated deterioration of the condition ${ }^{10)}$.

To our knowledge, there is a dearth of research into the actual management approaches aimed at addressing poor compliance with prescribed exercise programs in FMS. Strategies to increase compliance to effective treatment strategies such as exercise therapy in FMS are therefore warranted. The inference that pain catastrophization and subsequent fear-avoidance behaviors may influence the 
compliance of patients with FMS with exercise programs, and this justifies finding treatment approaches to alter pain catastrophization in the management of $\mathrm{FMS}^{18,19)}$. It is therefore postulated that by reducing pain catastrophization in patients with FMS, fear of movement would be decreased, compliance with exercise therapy would be increased, and the real physiological and psychological benefits of exercise therapy for patients with FMS would be realized.

Empirical evidence suggests that cognitive behavioural therapy (CBT), specifically exposure therapy, may be useful in the alteration of pain catastrophization observed in patients with FMS ${ }^{17)}$. Traditionally, exposure therapy is conducted during real situations (in vivo exposure therapy) or during an imagined situation (imagined exposure therapy). However, more recent innovations indicate that exposure therapy may also be administered via virtual reality technology, namely virtual reality exposure therapy (VRET) or in virtuo exposure therapy ${ }^{20,21)}$. VRET is a type of exposure therapy in which the user can be immersed into a computer-generated environment via a head-mounted display and visually exposed to a simulation of a specific feared situation ${ }^{20-22)}$. Contrary to other types of exposure therapy, VRET seems ideal for conditions in which the real situations are inaccessible or costly (in vivo exposure) or individuals find it difficult to imagine certain situations (imagined exposure therapy) ${ }^{20,21)}$. To date, VRET has successfully been used for other forms of phobias, such as fear of spiders and flying ${ }^{20-22)}$, but has never been used in the treatment of fear of movement/exercise, or for pain catastrophizing in chronic pain conditions.

Investigation of a novel VRET exercise program as a possible treatment option for pain catastrophizing in FMS is plausible because it has been shown that imagined exposure therapy may effectively reduce pain catastrophizing in patients with FMS ${ }^{17}$. However, since there is no available VRET exercise program for the treatment of pain catastrophizing in patients with FMS; preliminary steps were required prior to further development and testing of such a program. Initially, it had to be ascertained if visual exposure to catastrophized exercise activities cognitively triggered functional brain areas associated with pain catastrophizing in patients with FMS. The premise was that if visual stimuli of the catastrophized exercise activities cognitively triggered pain catastrophizing in previously identified functional brain areas of patients with FMS $^{23}$, a VRET program aimed at exposing patients with FMS to visuals of the feared or catastrophized exercises and neutralizing feelings of catastrophization towards exercise activities could possibly decrease pain catastrophizing and subsequently decrease fear of movement. In turn, compliance with prescribed exercise programs in clinical practice may be increased.

The following study was therefore primarily aimed at testing the novel concept that exposing patients with FMS (who catastrophized pain related to exercise), to healthy exercise activities presented via visuals elicits neurophysiological changes in functional brain areas associated with pain catastrophization. It was hypothesized that subjects with FMS, when compared with healthy matched controls, would display significant activation in functional areas associated with pain catastrophization when exposed to visuals of exercise activities.

\section{SUBJECTS AND METHODS}

Ethical approval for this study was initially obtained from the Health Research Ethics Committee of Stellenbosch University, South Africa, during July 2010. The study protocol was also approved by the Committee of Postgraduate Education of Stellenbosch University, the Cape Universities Brain Imaging Centre (CUBIC) research committee, and the Department of Health/Provincial Government of the Western Cape, South Africa. All eligible subjects were required to read and sign an informed consent form in their preferred language prior to participating in this study. This study was conducted at CUBIC and the Rheumatology clinic and Occupational Therapy Department of Tygerberg Hospital's (TBH). The study incorporated an exploratory, case-controlled study design.

A sociodemographic data form was designed by the principal researcher to collect sociodemographic information. Pain severity was assessed using a five-point pain severity scale (PSS). The South African Pain Catastrophizing Scale (SA-PCS) was used to measure pain catastrophizing. The South African Tampa Scale for Kinesiophobia (SA-TSK) was used to measure kinesiophobia (fear of movement). The South African Revised Fibromyalgia Impact Questionnaire (SA-FIQR) was used to measure the impact of fibromyalgia. The SA-PCS, SA-TSK and SA-FIQR had been previously cross-culturally adapted and validated among a group of patients with FMS living in and around the Cape Metropole area of the Western Cape, South Africa. The adapted versions of these outcome measures showed good psychometric properties $^{24)}$. Physical activity levels were assessed using the General Practice Physical Activity Questionnaire (GPPAQ). Neurophysiological observations were obtained using functional magnetic resonance imaging (fMRI).

Subjects were consecutively recruited from the available FMS population currently registered at the TBH Rheumatology Clinic, based on the following eligibility criteria: female adults aged 18 years and older, clinically diagnosed with FMS according to the American College Rheumatology (ACR) criteria by a qualified rheumatologist, registered as a patient at the TBH Rheumatology Clinic; South African citizens residing in or around the Cape Metropole area; scored $>24$ points on the SA-PCS and $>37$ points on the SATSK, and willing to undergo fMRI scanning. Subjects were excluded if they were diagnosed with any other conditions not related to FMS, i.e., cancer, HIV/AIDS, etc.; had severe physical disabilities; suffered from other chronic rheumatoid conditions, i.e., systemic lupus erythematosus, rheumatoid arthritis, etc.; suffered from psychological/psychiatric disorders, i.e., bipolar disorder, etc.; had previously been hospitalized for a major psychiatric disorder; had an uncontrolled endocrine/allergic disorder; were using medication other than the prescribed pharmacologic agents for FMS symptoms; were currently or had previously abused any illicit substances or alcohol; or were unable to discontinue intake of antidepressants four weeks prior to commencement of the study.

Controls were purposefully selected and matched based on age, race, gender, and socioeconomic status and were recruited from areas surrounding the $\mathrm{TBH}$ area. Controls 
were included if they were healthy and did not possess any chronic rheumatoid conditions, i.e., FMS, systemic lupus erythematosus, rheumatoid arthritis, etc.; any medical/psychological or psychiatric conditions, i.e., cancer, HIV/AIDS, bipolar disorder or clinical depression, etc.; or any physical disabilities.

In addition, both the subjects and controls were excluded if they displayed any contraindications that prohibited the use of fMRI, i.e., cardiac pacemakers, metal implants, claustrophobia, pregnancy, cochlear implants, etc.; or if they had a bust/chest size of more than $1.5 \mathrm{~m}$ in circumference since, the MRI scanner at CUBIC did not cater for larger bust/chest sizes.

A simple block design was used for the fMRI task, which allowed for simple modelling of the blood-oxygenationlevel-dependent (BOLD) response, resulting in more robust and reproducible results ${ }^{25)}$. The fMRI visual task was designed in the E-Studio 2.0.8.90 (E-Prime 2.0) software available at the CUBIC facility using the selected visuals. The fMRI visual task consisted of the following two stimuli: i) active visuals (clips of various visuals of exercise/physical activities, i.e., cycling, running, etc.) and ii) passive visuals (clips of visuals of everyday sedentary/relaxing activities, i.e., reading a book/magazine, drinking tea, etc.). The fMRI task was set up to include twelve $20 \mathrm{~s}$ "off"/rest periods (no stimulus) and twelve $30 \mathrm{~s}$ "on" periods (stimuli). The "off"/ rest period comprised a fixation visual (a small grey block in the middle of a black screen). The total duration of the task was $600 \mathrm{~s}$ (10 minutes). The $30 \mathrm{~s}$ "on" periods consisted of either six active visuals (active condition) or six passive visuals (passive condition). Each visual was flashed for $5 \mathrm{~s}$. The active visuals, passive visuals, and rest period were alternated. The design of the fMRI task model was as follows: $r A r B r A r B r A r B r A r B r A r B r A r B$, where $A=$ active condition, $B=$ passive condition, and $r=$ rest period. In total, from preparation to completion, the scanning procedure for each subject lasted approximately $40 \mathrm{~min}$ (15 min for preparation, plus 9 min for a multi-echo MPRAGE (MEMPR structural) scan, plus $10 \mathrm{~min}$ for the fMRI task and approximately $5 \mathrm{~min}$ extra time).

On the day of the scheduled fMRI scanning, subjects/ controls were escorted to the fMRI chamber room and asked to lie down inside the fMRI chamber. Foam cushions were used to immobilize the subject's/control's head. Subjects/ controls were required to wear MRI-compatible earplugs (to minimize scanner noise) and earmuffs (for communication with the radiographer/principle researcher during the scanning process). A pilot study was conducted to test the logistics (including the duration, applicability and acceptability) of the fMRI visual task and procedure. One subject clinically diagnosed with FMS and meeting the subject inclusion criteria participated in the pilot study.

Standard MPRAGE (MEMPR structural brain scans) and blood-oxygenation-level-dependant (BOLD) sequences were acquired using a Siemens MAGNETOM Allegra 3 Tesla (3T) MRI scanner (Siemens, Munich, Germany). T1weighted structural images interleaved in a sagittal direction and a isocenter position were acquired using a multi-echo MEMPRAGE sequence ${ }^{26)}$ with the following parameters: an echo time (TE) of $1.53 \mathrm{~ms}$, recovery time (TR) of $2.53 \mathrm{~ms}$, flip angle of $7^{\circ}, 256 \times 256$ pixel matrix, field of vision (FOV) of $256 \mathrm{~mm}, 1.3 \times 1 \times 1.3 \mathrm{~mm}$ voxels, and 128 partitions per slab. This structural scanning session was followed by two functional scan sessions using multi-slice, echo-planar imaging fMRI acquisition with the following parameters: a TE of $23 \mathrm{~ms}$, TR of $1.6 \mathrm{~s}$, flip angle of $73^{\circ}, 64 \times 64$ pixel matrix, FOV of $255 \mathrm{~m}, 30$ horizontal $3 \mathrm{~mm}$ slices. These parameters allowed coverage of the entire brain with $4 \mathrm{~mm}^{3}$ voxels within $5 \mathrm{~s}$. During each fMRI session, the first four scans were discarded to allow for saturation of the tissue. Starting on the fourth scan, $20 \mathrm{~s}$ rest periods ("off" condition) were alternated with $30 \mathrm{~s}$ stimuli ("on" condition/active or passive visuals). Analysis was performed on the scans acquired under the "on" and "off" conditions. BOLD contrasts were acquired during the fMRI scans for the subject under the following conditions: 1) active $>$ rest condition (where brain activations during the rest period were subtracted from those during the active visuals); 2) passive $>$ rest condition (where brain activations during the rest period visuals were subtracted from those during the passive visuals); 3 ) active $>$ passive condition (where brain activations during passive condition were subtracted from those during the active visuals); and 4) passive $>$ active condition (where brain activations during active visuals were subtracted from those during the passive visuals).

Data acquired from the subjects were preprocessed by the principal researcher using Oxford's FMRIB Software Library (FSL), a collection of functional and structural brain image analysis tools, written by members of the Image Analysis Group at the Oxford Centre for Functional Magnetic Resonance Imaging of the Brain, Oxford University ${ }^{27}$. FSL standard routines and templates were used for preprocessing of functional MRI data: realignment, normalization (resulting voxel size $2 \times 2 \times 2 \mathrm{~mm}$ ), and smoothing ( $8 \mathrm{~mm}$ isotropic Gaussian kernel; high-pass filter cutoff of $100 \mathrm{~s}$ ). Each session of image acquisition was defined as a separate session in the realignment procedure. Head motion was determined by motion detection software and visual inspection of raw and processed images. Head motion greater than half a voxel was deemed a priori to be unacceptable, and images meeting this criterion were excluded. None of the scans acquired in this study had head motion exceeding this criterion, so all images were included in the analyses.

After preprocessing the data, individual subject data analysis was performed by linear regression of the fMRI data in FSL. The preprocessed data were assigned to the following two conditions in the model specification: the active condition and passive condition. For each of the participants, four BOLD contrast differences (t-contrasts) were determined as a function of BOLD signal changes compared with the baseline condition (active $>$ rest; passive $>$ rest; active $>$ passive; passive $>$ active) according to the routine procedures implemented. The brain volumes collected during the "on" conditions were compared with the brain volumes collected during the "off" conditions using the Student's ttest. Resultant $\mathrm{Z}$ statistical volumes and mean differences volumes were registered into standardized space using the statistical parametric mapping (FSL) echo-planar imaging template and re-sliced to $2 \mathrm{~mm}^{3}$ voxels. Anatomic regions were identified (i) by inspection of individual functional 
images superimposed on an individual's structural image, and (ii) by conversion of the coordinates to the coordinate system of the Montreal Neurological Institute (MNI) atlas and localization using this atlas and automated software. The scans were registered as high resolution MNI structural scans so that the FSL atlas tools could be used to correctly locate these regions of interest (ROIs). Statistic images were thresholded using clusters determined by $\mathrm{Z}>2.3$ and a (corrected) cluster significance level of $\mathrm{p}=0.05$.

Higher-level analyses of the subject fMRI data were also conducted in FSL. Mean activation of functional was compared within and between groups of FMS subjects and the healthy controls. Anatomical localization of activated brain regions was determined by reference to the atlas tool automatically implemented in FSL. The paired t- test was used for comparison of two dependent samples and contrasts. Statistic images were thresholded using clusters determined by $\mathrm{Z}>2.3$ and a (corrected) cluster significance level of $\mathrm{p}=0.05$.

\section{RESULTS}

A total of 13 subjects with FMS and nine matched healthy controls participated in this study. In Table 1, a comparison of the sociodemographic information for the FMS subject group and control group is depicted. One FMS subject withdrew from the study shortly before the fMRI scans took place. Baseline demographic data were, however, included for this subject along with the rest of the subjects (Table 1). All subjects and controls lived in the surrounding areas of the Cape Metropole. There were no significant differences $(p>0.05)$ in age or number of children between the FMS subject group and the matched control group. There was, however, a significant difference in the number of physically active hours per week $(\mathrm{p}<0.0000)$ between the two study groups (Table 1)

The mean difference in activation of the functional brain areas between the two groups, the FMS subject group and the matched control group, was analyzed. The images from the functional data analyses in Fig. 1 illustrates significant differences $(p<0.05)$ in functional brain area activation for the active $>$ passive condition between the FMS subject group and the matched control group.

In Table 2, the $\mathrm{Z}$ co-ordinates ( $\mathrm{x}, \mathrm{y}$ and $\mathrm{z}$ ), Z-scores as well as $p$-values for all the areas significantly activated $(p<0.05)$ during the active $>$ passive condition for FMS subject group $>$ matched control group, where brain activations acquired for the matched control group were subtracted from the brain activations acquired for the FMS subject group during the active>passive condition.

\section{DISCUSSION}

The current paper reports on the first investigation (to our knowledge) into proving the novel concept that exposing patients with FMS to healthy exercise activities via visuals elicits neurophysiological changes in functional brain areas associated with pain catastrophization. Preliminary support was provided for the further development and testing of a VRET exercise program aimed at reducing pain catastrophization related to exercise therapy in patients with FMS.
Table 1. Baseline comparison of sociodemographic characteristics of FMS subjects and matched controls

\begin{tabular}{|c|c|c|}
\hline Variable & FMS subjects & Matched Controls \\
\hline & $\mathrm{N}=13$ & $\mathrm{~N}=9$ \\
\hline Gender & Female & Female \\
\hline Age (yrs) mean \pm SD & $46.00 \pm 9.72$ & $48.22 \pm 14.77$ \\
\hline Number of children & $2.75 \pm 1.14$ & $2.75 \pm 0.88$ \\
\hline \multicolumn{3}{|l|}{ Marital status } \\
\hline Single & 2 & 1 \\
\hline Married & 6 & 5 \\
\hline Separated & 2 & 0 \\
\hline Divorced & 2 & 2 \\
\hline Widowed & 1 & 1 \\
\hline $\begin{array}{l}\text { Years living with FMS } \\
\text { mean } \pm \text { SD }\end{array}$ & $3.81 \pm 2.27$ & NA \\
\hline \multicolumn{3}{|l|}{ Ethnicity } \\
\hline Colored & 9 & 6 \\
\hline Black & 4 & 3 \\
\hline White & 0 & 0 \\
\hline \multicolumn{3}{|l|}{ Language } \\
\hline English & 2 & 1 \\
\hline Afrikaans & 6 & 5 \\
\hline Xhosa & 4 & 3 \\
\hline \multicolumn{3}{|l|}{ Level of education } \\
\hline$<$ grade 7 & 5 & 4 \\
\hline$>$ grade 12 & 4 & 2 \\
\hline Matric & 3 & 2 \\
\hline Tertiary education & 1 & 1 \\
\hline \multicolumn{3}{|l|}{ Employment status } \\
\hline Unemployed & 6 & 1 \\
\hline Permanently employed & 0 & 5 \\
\hline Self-employed & 2 & 0 \\
\hline Casually employed & 1 & 0 \\
\hline Pensioner & 1 & 1 \\
\hline Housewife & 2 & 2 \\
\hline Disability grant & 1 & 0 \\
\hline $\begin{array}{l}\text { Physical activity hours/ } \\
\text { week mean } \pm \text { SD }\end{array}$ & $9.62 \pm 6.79$ & $39.44 \pm 12.61$ \\
\hline
\end{tabular}

NA: not applicable, SD: standard deviation, *significant $(\mathrm{p}<0.05)$

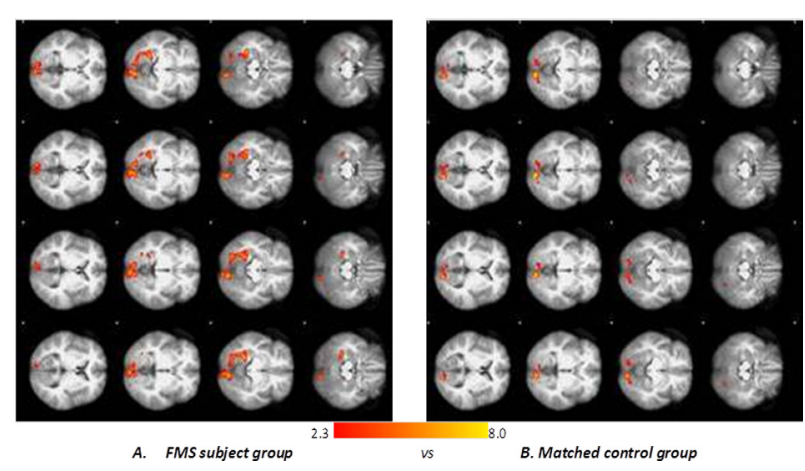

Fig. 1. Between-group comparison of significant activation $(p<0.05)$ of functional areas in the active>passive condition 
Table 2. Significant differences in functional area activation for FMS subjects $>$ matched controls contrast during the active $>$ passive condition

\begin{tabular}{lrrrrr}
\hline \multirow{2}{*}{ Functional area } & \multicolumn{3}{c}{ Co-ordinates MNI } & \\
& \multicolumn{5}{c}{ (FSL) } \\
\hline & $\mathrm{x}$ & $\mathrm{y}$ & $\mathrm{z}$ & \\
\hline Right inferior temporal gyrus, right cerebellum posterior lobe & 47 & -61 & -22 & $*$ \\
Right inferior and middle frontal gyrus & 42 & 10 & 23 & $*$ \\
Left inferior frontal gyrus & -44 & 24 & 14 & $*$ \\
Left superior parietal lobe, L Supramarginal gyrus & -29 & 54 & 53 & $*$ \\
Left thalamus & -23 & -29 & 9 & $*$ \\
\hline
\end{tabular}

MNI: Montreal Neurological Institute, FSL: Oxford's FMRIB Software Library, *significant $(\mathrm{p}<0.05)$

The study findings indicated that the FMS subject group showed significant activation in the right and left inferior frontal gyri $(\mathrm{p}<0.0000)$ in the active $>$ passive condition when compared with the healthy controls. These results concur with a study conducted by Gracely et al., who found that activity in the inferior frontal gyrus was associated with pain catastrophization in high catastrophizing patients with FMS $^{23)}$. More recently, however, studies have examined the role of the inferior frontal cortex in belief-bias reasoning and deductive reasoning tasks using repetitive transcranial magnetic stimulation (rTMS) ${ }^{28,29)}$. The studies found that the inferior frontal gyrus may play a significant role in belief-bias reasoning and deductive reasoning tasks ${ }^{28,29)}$ Belief bias is currently defined as the tendency of an individual to be "erroneously biased when logical conclusions are incongruent with belief about the world" ${ }^{28)}$. Although the definition of belief-bias reasoning is different from that of pain catastrophizing, the significant activation of the right and left inferior frontal gyri in the FMS subjects, but not the controls, in the active> passive condition in this study implies that there may have been a tendency for the FMS subjects to have a belief-bias reasoning reaction in response to the exercise activities similar to the catastrophization reaction of the subjects in response to exercise activities. Although the study conducted by Gracely et al. differed considerably from the current study in terms of the stimuli used during the fMRI task, the fact that the right and left inferior frontal gyri were exclusively activated in the FMS subject group may provide further support for the involvement of the inferior frontal gyri in pain catastrophization and belief-bias reasoning related to non-painful stimuli. However, further research is required to ascertain the exact involvement of the inferior frontal gyri in pain catastrophization and belief-bias reasoning in patients with FMS.

Right posterior cerebellum activation was also significant $(p<0.000)$ for the FMS subject group in the active $>$ passive condition when compared with the healthy controls. The cerebellum plays an important role in motor control but may also be involved in some cognitive functions such as attention and language, and affective regulation, such as regulating of fear and pleasure responses ${ }^{30,31}$. Previously it was also reported that viewing emotional images from the International Affective Picture Scale (IAPS) activates the posterior lobe of the cerebellum when compared with viewing neutral images ${ }^{32}$. Activation of the cerebellum was also seen in neuroimaging studies investigating panic, as well as sadness and grief ${ }^{31)}$. The posterior cerebellum has also been found to be activated during painful stimulation but to be more specifically activated during the anticipation of pain ${ }^{33)}$. Furthermore, studies have also found that the posterior cerebellar regions are involved when processing one's own painful experiences ${ }^{31)}$ and may also be associated with pain catastrophization in high catastrophizing FMS patients $^{23}$. The functions currently reported for the posterior cerebellum can possibly explain the significant activation of the posterior cerebellum when the subjects were exposed to visuals of exercise activities in the active $>$ passive condition. Although we cannot be certain if the activation of the posterior cerebellum was exclusively associated with pain catastrophization related to the exercise activities depicted in the active visuals, we cannot definitively discard this finding. Regardless, the posterior cerebellum is also reportedly involved in a variety of emotional processes, such as fear $^{30,31)}$, and these processes may explain the activation of this functional brain area in the active>passive condition in the FMS subject group, and lack of activation in the control group. The FMS subject group may have experienced feelings of fear, if not catastrophization, in response to the visuals depicting the exercise activities. The involvement of the posterior cerebellum in the processing of pain catastrophization and fear in response to exercise activities in patients with FMS needs to however be confirmed.

Significant activation $(\mathrm{p}<0.0000)$ in the right middle frontal gyrus was also found in the FMS subject group in the active $>$ passive condition. The middle frontal gyrus is involved in high-level executive functions and decisionrelated processes such as cognitive control, working memory, semantic processing, target detection, memory retrieval, recognition, prospective memory, and processing of emotional stimuli ${ }^{34)}$. In addition, it has also been reported that the middle frontal gyrus may be associated with pain catastrophization in high catastrophizing FMS patients ${ }^{23}$. These functions reported for the middle frontal gyrus may explain activation of this area in the FMS subject group in the active condition. The FMS group would have most probably seen the active visuals, recognized what the visuals were depicting (recognition), retrieved a memory related to that particular activity depicted in the visuals (memory 
retrieval), and then either processed an emotion in response to that particular activity depicted in the visuals (processing of emotional stimuli) or thought of what they would feel if they performed the activity (prospective memory); they may even have catastrophized in response to the exercise activities. Although this reaction to the active visuals cannot be stated for certain, it does provide a logical explanation for the activation of the middle frontal gyrus in the FMS subject group during the active $>$ passive condition in this study. The role of the middle frontal gyrus in processing emotions and constructs such as pain catastrophization in response to exercise activities in patients with FMS, however, should be further investigated.

According to Russ et al., the supramarginal gyrus, in addition to the middle and superior gyrus and the postcentral gyrus, may be involved in the enactment effect of memory ${ }^{35)}$. The enactment effect of memory is basically "learning by doing" or "encoding by performing", and it refers to the fact that performing a task verbally provided improves subsequent memory performance ${ }^{35}$. Despite the involvement of the other mentioned areas, the supramarginal gyrus, however, was believed to play a central role in this function ${ }^{35)}$. There are, however, various steps in enactment of memory procedures ${ }^{35)}$, and since the FMS subjects did not enact the activity they were exposed to during the fMRI scan to memorize it, they may have elicited activation of the left supramarginal gyrus in preparation for enacting what was being shown. However, this is pure speculation, and further research is warranted to ascertain the role of the supramarginal gyrus when an individual is exposed to visuals of various activities.

Since the inferior frontal gyrus, posterior cerebellum, and middle frontal gyrus have been previously associated with pain catastrophization in patients with $\mathrm{FMS}^{23}$ ), the findings of this study may indicate that subjects with FMS catastrophized the exercise activity visuals and not the passive visuals. However, it cannot be stated for certain whether the activation of these areas was solely associated with pain catastrophization in the patients with FMS due to one of their other reported functions of these areas. Nonetheless, the findings of this study provide preliminary support for the testing of a novel VRET exercise program as a treatment for pain catastrophization in patients with FMS. It is however recommended that further research be conducted to investigate the roles of the inferior frontal gyrus, posterior cerebellum, and middle frontal gyrus, as well as the amygdala, in processing pain catastrophization in response to exercise activities.

Although every effort was made to ensure that the visuals included in the final fMRI task were the most appropriate to elicit the construct of interest, i.e., pain catastrophization, the development of an fMRI task may require much more than was possible in this study. Time and financial constraints, however, prohibited us from extending the validation of the fMRI task indefinitely. There is, therefore, the possibility that subjects may have become bored or may have ceased to pay attention to the task for the entire duration of the scan, which could have influenced the activations in the brain and led to incorrect results. We would recommend that the fMRI tasks used in this study be further validated in larger samples to ensure that the tasks are indeed optimal to achieve ac- curate results.

In conclusion, although it may be many years before an adequate management strategy for treating pain catastrophization in response to exercise therapy in FMS is successfully implemented in clinical practices across the globe, the findings of this research significantly add to the current body of knowledge regarding the possibility of objectively identifying cognitive behavioural strategies like pain catastrophization often observed among chronic pain patients within the physiotherapy practice scope. The results of this study also provide a rather promising suggestion that a program like VRET aimed at reducing pain catastrophization related to exercise activities among patients with FMS may be worth further investigation. The development of such a program may finally provide physiotherapists with a tool to address issues such as poor patient compliance in clinical practice, which often hinders the successful management of FMS and other chronic conditions. Physiotherapists would not only be involved in prescribing exercise therapy in the management of FMS but could also become more involved in changing existing negative thoughts (i.e., catastrophizing thoughts) that a patient may have towards exercises. Compliance with exercise programs among FMS sufferers could therefore be increased. This research significantly contributes to a new era of research relating to finding evidence for the further development of effective strategies that may aid in the successful implementation and maintenance of exercise programs and other treatment modalities among patients with FMS.

\section{ACKNOWLEDGEMENTS}

The authors would like to thank the patients with fibromyalgia registered at $\mathrm{TBH}$ who were willing to participate in this study and would like to acknowledge the following individuals for their assistance in ensuring the success of this study: Dr. B. Spottiswoode (previous head of CUBIC); Mrs. Q. Makwanda (research assistant/Xhosa translator); Dr. M. Manie, Dr. R. Du Toit, Mrs. D. Fortune and the rest of the staff at the TBH Rheumatology clinic; Ms T. Ahmed, Ms S. Hugo and Mrs. R. Pillay at TBH Occupational Therapy department; Mrs. L Klein, Mrs. N. Maroof, and Mrs. M. De Villiers at CUBIC; Dr. F. Robertson and Dr. A. Alhamud at the University of Cape Town, and Prof. S Seedat (head of Psychiatry department, Stellenbosch University).

\section{REFERENCES}

1) Brosseau L, Wells GA, Tugwell P, et al. Ottawa Panel Members: Ottawa Panel evidence-based clinical practice guidelines for aerobic fitness exercises in the management of fibromyalgia: part 1. Phys Ther, 2008, 88: 857-871. [Medline] [CrossRef]

2) Kelley GA, Kelley KS, Hootman JM, et al.: Exercise and global well-being in community-dwelling adults with fibromyalgia: a systematic review with meta-analysis. BMC Public Health, 2010, 10: 198. [Medline] [CrossRef]

3) Thomas EN, Blotman F: Aerobic exercise in fibromyalgia: a practical review. Rheumatol Int, 2010, 30: 1143-1150. [Medline] [CrossRef]

4) Kelley GA, Kelley KS, Jones DL: Efficacy and effectiveness of exercise on tender points in adults with fibromyalgia: a meta-analysis of randomized controlled trials. Arthritis (Egypt), 2011, 2011: 125485. [Medline]

5) Williams DA, Clauw DJ: Understanding fibromyalgia: lessons from the broader pain research community. J Pain, 2009, 10: 777-791. [Medline] [CrossRef] 
6) Jones KD, Liptan GL: Exercise interventions in fibromyalgia: clinical applications from the evidence. Rheum Dis Clin North Am, 2009, 35: 373 391. [Medline] [CrossRef]

7) Vural M, Berkol TD, Erdogdu Z, et al.: Evaluation of the effectiveness of an aerobic exercise program and the personality characteristics of patients with fibromyalgia syndrome: a pilot study. J Phys Ther Sci, 2014, 26: 1561-1565. [Medline] [CrossRef]

8) Oliver K, Cronan T: Predictors of exercise behaviors among fibromyalgia patients. Prev Med, 2002, 35: 383-389. [Medline] [CrossRef]

9) Sevimli D, Kozanoglu E, Guzel R, et al.: The effects of aquatic, isometric strength-stretching and aerobic exercise on physical and psychological parameters of female patients with fibromyalgia syndrome. J Phys Ther Sci, 2015, 27: 1781-1786. [Medline] [CrossRef]

10) Gowans SE: Fibromyalgia: increased regular physical activity as 'exercise' in fibromyalgia. Nat Rev Rheumatol, 2010, 6: 499-500. [Medline] [CrossRef]

11) Genc A, Tur BS, Aytur YK, et al.: Does aerobic exercise affect the hypothalamic-pituitary-adrenal hormonal response in patients with fibromyalgia syndrome? J Phys Ther Sci, 2015, 27: 2225-2231. [Medline] [CrossRef]

12) Sullivan M, Bishop S: The pain catastrophizing scale: development and validation. Psychol Assess, 1995, 7: 524-532. [CrossRef]

13) Hassett AL, Cone JD, Patella SJ, et al.: The role of catastrophizing in the pain and depression of women with fibromyalgia syndrome. Arthritis Rheum, 2000, 43: 2493-2500. [Medline] [CrossRef]

14) Edwards RR, Bingham CO 3rd, Bathon J, et al.: Catastrophizing and pain in arthritis, fibromyalgia, and other rheumatic diseases. Arthritis Rheum, 2006, 55: 325-332. [Medline] [CrossRef]

15) Quartana PJ, Campbell CM, Edwards RR: Pain catastrophizing: a critical review. Expert Rev Neurother, 2009, 9: 745-758. [Medline] [CrossRef]

16) Börsbo B, Gerdle B, Peolsson M: Impact of the interaction between selfefficacy, symptoms and catastrophising on disability, quality of life and health in with chronic pain patients. Disabil Rehabil, 2010, 32: 1387-1396. [Medline] [CrossRef]

17) Rodero B, García J, Casanueva B, et al.: [Imagined exposure as treatment of catastrophizing in fibromyalgia: a pilot study]. Actas Esp Psiquiatr, 2008, 36: 223-226. [Medline]

18) Alda M, Luciano JV, Andrés E, et al.: Effectiveness of cognitive behaviour therapy for the treatment of catastrophisation in patients with fibromyalgia: a randomised controlled trial. Arthritis Res Ther, 2011, 13: R173. [Medline] [CrossRef]

19) van Koulil S, Effting M, Kraaimaat FW, et al.: Cognitive-behavioural therapies and exercise programmes for patients with fibromyalgia: state of the art and future directions. Ann Rheum Dis, 2007, 66: 571-581. [Medline] [CrossRef]

20) Parsons TD, Rizzo AA: Affective outcomes of virtual reality exposure therapy for anxiety and specific phobias: a meta-analysis. J Behav Ther Exp Psychiatry, 2008, 39: 250-261. [Medline] [CrossRef]

21) Powers MB, Emmelkamp PM: Virtual reality exposure therapy for anxiety disorders: a meta-analysis. J Anxiety Disord, 2008, 22: 561-569. [Medline] [CrossRef]

22) Bush J: Viability of virtual reality exposure therapy as a treatment alternative. Comput Human Behav, 2008, 24: 1032-1040. [CrossRef]

23) Gracely RH, Geisser ME, Giesecke T, et al.: Pain catastrophizing and neural responses to pain among persons with fibromyalgia. Brain, 2004, 127: 835-843. [Medline] [CrossRef]

24) Morris LD, Grimmer-Somers KA, Louw QA, et al.: Cross-cultural adaptation and validation of the South African Pain Catastrophizing Scale (SAPCS) among patients with fibromyalgia. Health Qual Life Outcomes, 2012, 10: 137. [Medline] [CrossRef]

25) Amaro E Jr, Barker GJ: Study design in fMRI: basic principles. Brain Cogn, 2006, 60: 220-232. [Medline] [CrossRef]

26) van der Kouwe AJ, Benner T, Salat DH, et al.: Brain morphometry with multiecho MPRAGE. Neuroimage, 2008, 40: 559-569. [Medline] [CrossRef]

27) Smith SM, Jenkinson M, Woolrich MW, et al.: Advances in functional and structural MR image analysis and implementation as FSL. Neuroimage, 2004, 23: S208-S219. [Medline] [CrossRef]

28) Tsujii $T$, Masuda $S$, Akiyama $T$, et al.: The role of inferior frontal cortex in belief-bias reasoning: an rTMS study. Neuropsychologia, 2010, 48: 2005-2008. [Medline] [CrossRef]

29) Tsujii T, Sakatani K, Masuda S, et al.: Evaluating the roles of the inferior frontal gyrus and superior parietal lobule in deductive reasoning: an rTMS study. Neuroimage, 2011, 58: 640-646. [Medline] [CrossRef]

30) Baillieux H, De Smet HJ, Paquier PF, et al.: Cerebellar neurocognition: insights into the bottom of the brain. Clin Neurol Neurosurg, 2008, 110. 763-773. [Medline] [CrossRef]

31) Stoodley CJ, Schmahmann JD: Evidence for topographic organization in the cerebellum of motor control versus cognitive and affective processing. Cortex, 2010, 46: 831-844. [Medline] [CrossRef]

32) Lang $P$, Bradley $M$, Cuthbert $B$ : International affective picture system (IAPS): Affective ratings of pictures and instruction manual. Gainsville: University of Florida, Technical Report 2005.

33) Ploghaus A, Tracey I, Gati JS, et al.: Dissociating pain from its anticipation in the human brain. Science, 1999, 284: 1979-1981. [Medline] [CrossRef]

34) Talati A, Hirsch J: Functional specialization within the medial frontal gyrus for perceptual go/no-go decisions based on "what," "when," and "where" related information: an fMRI study. J Cogn Neurosci, 2005, 17: 981-993. [Medline] [CrossRef]

35) Russ MO, Mack W, Grama CR, et al.: Enactment effect in memory: evidence concerning the function of the supramarginal gyrus. Exp Brain Res, 2003, 149: 497-504. [Medline] 